\title{
A novel nanoscaled vertical hybrid GaN HEMT
}

\author{
Sumit Verma ${ }^{1}$, Humyra Shabir ${ }^{1}$ and Sajad A Loan ${ }^{1 *}$ and A. R. M Alamoud ${ }^{2}$ \\ 1Department of Electronics and Communication Engineering, Jamia Millia Islamia, New Delhi- \\ 110025 2Department of Electrical Engineering, King Saud University, Saudi Arabia \\ *Email: sajadiitk@gmail.com
}

The increasing demand of highly efficient, high speed and high power electronic devices has posed a big challenge to device designers to extract these features for the current solution, that is, silicon. Since the silicon based power devices have reached their theoretical limit in terms of high power, high frequency, and high temperature operations by virtue of the physical properties of silicon. The further enhancement in performance in silicon based device is incremental, however, the cost is becoming increasingly uneconomical. Therefore, new materials and new devices are needed, which can fulfill the demand of high performance of future electronic devices. The semiconductor, which can fulfill the requirements of high frequency, high power and high temperature operation for the third generation of wireless and communication technology, is the Gallium Nitride $(\mathrm{GaN})$. GaN and its alloys are widely acknowledged as primary candidates for high power microwave applications due to their high breakdown voltage $(3 \mathrm{MV} / \mathrm{cm})$, high electron saturation velocity $\left(2.5 \times 10^{7} \mathrm{~cm} / \mathrm{sec}\right)$ and high operating temperatures.

Two types of GaN structures that are being investigated are lateral and vertical. Most of the work available in literature is concentrated on lateral device topology because they are easy to fabricate. To enhance the breakdown voltage for lateral HEMTs gate and drain separations are usually increased. But this make the chip size larger. In contrast, to enhance the breakdown voltage in vertical structures just leads to an increase in the buffer layer thickness. Thus vertical topology are expected to achieve a higher power density over lateral HEMTs for high breakdown voltage devices. Vertical geometry is also favored for ease of packaging and will also results in reduction in wafer area. Traditional $\mathrm{AlGaN} / \mathrm{GaN}$ vertical HEMT consist of $2 \mathrm{DEG}$ at $\mathrm{AlGaN} / \mathrm{GaN}$ interface on top and doped $\mathrm{N}+\mathrm{GaN}$ layer at bottom for making drain contact. Thus they are not dopingless in true sense. Also the thermal budget is high and $\mathrm{N}^{+}$doped GaN layer can result in random doping fluctuations. Here we proposed charge plasma based vertical hybrid GaN HEMT. In the proposed device, the $\mathrm{N}^{+}$ drain region is formed using the concept of charge plasma instead of traditional doping. Thus the proposed structure have low thermal budget.

The proposed structure is shown in Figure 1. The structure consist $15 \mathrm{~nm} \mathrm{AlGaN}$ layer at top. The $\mathrm{Al}$ composition in $\mathrm{AlGaN}$ layer is taken as 0.24 . GaN layer thickness is $3.49 \mu \mathrm{m}$. Again, 10 $\mathrm{nm}$ undoped GaN (UD-GaN) layer is taken at bottom to form drain region. The drain electrode is with work function of $3.9 \mathrm{eV}$ made from Hafnium metal has been used to induce $n^{+}$ charge plasma's in undoped GaN film to create drain region. The $\mathrm{P}-\mathrm{GaN}$ current blocking layer doping is $5 \times 10^{16}$. The aperture length $\left(\mathrm{L}_{\mathrm{A}}\right)$ is $0.8 \mu \mathrm{m}$ and gate length $\left(\mathrm{L}_{\mathrm{G}}\right)$ is $3 \mu \mathrm{m}$.

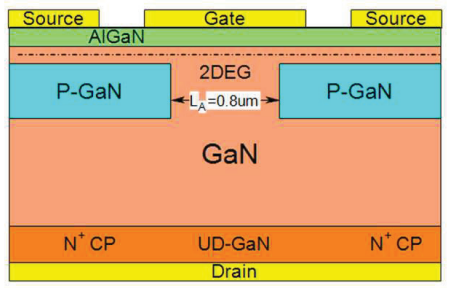

Figure 1: Schematic of proposed device

The behavior of proposed device is studied using Atlas. The input characteristics are shown and the transconductance have been shown in Figure $2 \mathrm{a}$ and $2 \mathrm{~b}$, respectively.
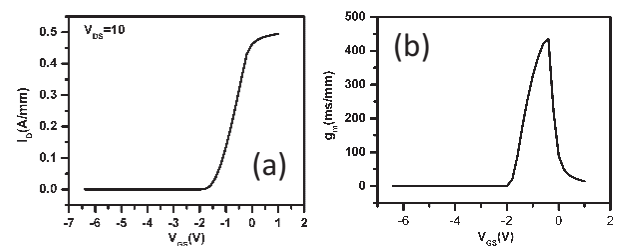

Figure 2: (a) Input Characteristics and (b) Transconductance of the proposed device 